\title{
Note
}

\section{Disruption of transforming growth factor- $\beta$ signaling in thyroid follicular epithelial cells or intrathyroidal fibroblasts does not promote thyroid carcinogenesis}

\author{
Mika Shimamura $^{1)}$, Mami Nakahara ${ }^{1)}$, Tomomi Kurashige ${ }^{1)}$, Kazuaki Yasui ${ }^{1)}$, Masahiro Nakashima ${ }^{2)}$ and \\ Yuji Nagayama ${ }^{1)}$ \\ 1) Department of Molecular Medicine, Atomic Bomb Disease Institute, Nagasaki University, Nagasaki 852-8523 Japan \\ 2) Department of Tumor and Diagnostic Pathology, Atomic Bomb Disease Institute, Nagasaki University, Nagasaki 852-8523 Japan
}

\begin{abstract}
Transforming growth factor $\beta$ (TGF- $\beta$ ) members, pleiotropic cytokines, play a critical role for carcinogenesis generally as a tumor suppressor in the early cancer development, but as a tumor promoter in the late stage of cancer progression. The present study was designed to clarify the role for TGF- $\beta$ signaling in early thyroid carcinogenesis using the conditional Tgfbr $2^{\text {floxE2/floxE2 }}$ knock-in mice, having 2 loxP sites at introns 1 and 2 of Tgfb2r gene. When these mice were crossed with thyroid peroxidase (TPO)-Cre or fibroblast-specific protein-1 (FSP1)-Cre, the resultant mice, Tgfbr $2^{t p o K O}$ and Tgfbr ${ }^{f s p K O}$, lost TGF- $\beta$ II receptor expression (thereby TGF- $\beta$ signaling) specifically in the thyroid follicular epithelial cells or fibroblasts, respectively. The thyroid morphology was monitored up to 52 weeks in these mice, showing no tumor development, except one Tgfbr $2^{t p o K O}$ mouse developing follicular adenoma like-lesion. Our data suggest that TGF- $\beta$ signaling in mesenchymal or follicular epithelial cells of the thyroid does not appear to function as a tumor suppressive barrier at the early stage of thyroid carcinogenesis.
\end{abstract}

Key words: TGF- $\beta$, Thyroid cancer, Epithelial cells, Fibroblasts

TRANSFORMING GROWTH FACTOR $\boldsymbol{\beta}$ (TGF- $\beta$ ) members are ubiquitously expressed multifunctional cytokines, and play a pivotal role in cancer development and progression [1]. It is generally recognized that TGF- $\beta$ is a tumor suppressor in the early carcinogenesis, but is a tumor promoter in the late stage of cancer progression $[2,3]$.

TGF- $\beta$ binds to TGF- $\beta$ type II receptor (T $\beta$ RII) and subsequently recruits the type I receptor (TRRI) for downstream cytoplasmic signaling through multiple parallel signaling pathways including the Smad proteins [1]. Although the significance of TGF- $\beta$ in cancer development/progression can be studied in vitro by using TGF- $\beta$ ligands and/or inhibitors for the T $\beta R$, genetically engineered Tgfbr2 $2^{\text {floxE2/floxE2 }}$ knock-in mice, having 2 lox $\mathrm{P}$ sites at introns 1 and 2 of $T g f \beta r 2$

Submitted Nov. 9, 2013; Accepted Nov. 27, 2013 as EJ13-0475

Released online in J-STAGE as advance publication Dec. 10, 2013

Correspondence to: Yuji Nagayama, M.D., Department of Molecular Medicine, Atomic Bomb Disease Institute, Nagasaki University, 1-12-4 Sakamoto, Nagasaki 852-8523 Japan.

E-mail: nagayama@nagasaki-u.ac.jp

(c) The Japan Endocrine Society (the gene encoding T $\beta$ RII) and conditionally inactive for $T g f b r 2$ in the presence of Cre DNA recombinase, have been shown to be highly valuable for in vivo studies for this purpose [4].

When these mice were crossed with fibroblast specific protein-1 (FSP1)-Cre mice in which Cre recombinase was specifically expressed in fibroblasts by the transcriptional control of the FSP-1 promoter, the resultant Tgfbr $2^{f s p K O}$ mice lost TGF- $\beta$ signaling exclusively in fibroblasts and developed intraepithelial neoplasia in prostate and invasive squamous cell carcinoma of the forestomach by 7 weeks of age, both associated with increased abundance of stromal cells [4]. Development of invasive tumors in forestomach and a loss of parietal cells in the glandular stomach were thought to be the cause of early death ( $\sim 8$ weeks of age) in these mice. The former prostate tumors progressed to overt adenocarcinomas by 7 months when transplanted into immune-compromised mice [5]. Furthermore, fibroblasts from these mice promoted growth, invasion and metastasis of mammary carcinoma [6, 7].

On the other hand, in parenchymal cells, crossing 
of Tgfbr $2^{\text {floxE2/floxE2 }}$ mice with mouse mammary tumor virus (MMTV)-Cre mice resulted in mice disrupted TGF- $\beta$ signaling specifically in mammary epithelial cells, in which development of mammary hyperplasia was observed [8]. Furthermore, the combination with the MMTV-polyomavirus middle T antigen transgenic PyVmt mice, which develop mammary cancer by themselves, resulted in shortened latency of cancer development and increased pulmonary metastasis. In another study, Tgfbr $2^{I E K O}$ mice (generated by crossing Tgfbr $2^{f l o x E 2 / f l o x E 2}$ and Villi-Cre mice, leading to intestine-specific T $\beta R I I$ loss) did not develop intestinal tumors by themselves, but when combined with Kras ${ }^{G 12 D}$ knock-in mice developed intestinal neoplasia and metastasis [9] and when combined with $\mathrm{APC}^{1638 N /}$ ${ }^{w t}$ mice developed more advanced intestinal tumors as compared to $\mathrm{APC}^{1638 N / w t}$ mice [10]. Thus, both mesenchymal and epithelial TGF- $\beta$ signaling plays crucial roles for early carcinogenesis process of some tissues as a tumor suppressor.

In the thyroids, as for other tissues, expression of TGF- $\beta$ ligands and their receptors and their effects on growth of normal cells and established cancer cells have previously been studied [11, 12], but yet the effect of TGF- $\beta$ signaling on early thyroid carcinogenesis remained to be determined. In this current study, therefore, Tgfbr $2^{\text {floxE2 ffloxE2 }}$ mice were crossed with FSP1Cre or thyroid peroxidase (TPO)-Cre mice to study the significance of TGF- $\beta$ signaling in thyroid follicular epithelial cells and surrounding fibroblasts for thyroid early carcinogenesis.

\section{Materials and Methods}

\section{Generation and characterization of Tgfbr $2^{\text {floxE2/floxE2, }}$ TPO-Cre, FSP1-Cre, Tgfbr2 $2^{f s p K O}$ and Tgfbr $2^{\text {tpoKO }}$ mice and experimental designs}

Tgfbr $2^{\text {floxE2/floxE2 } 2}$ knock-in mice have 2 loxP sites at introns 1 and 2 of Tgfbr2 [4]. Transgenic FSP1-Cre mice express Cre recombinase under the control of FSP-1 promoter [4]. FSP-1 is selectively expressed in fibroblasts. These 2 genetically engineered mice were kindly provided by Moss HL (Vanderbilt University). Transgenic TPO-Cre mice expressing Cre under the control of TPO promoter, a thyroid-specific promoter, were also previously generated and a kind gift from Kimura S (NIH) [13].

Tgfbr $2^{\text {floxE2/floxE2 }}$ mice were crossed with FSP1-Cre mice, generating Tgfbr $2^{\text {floxE2/wt }}$ FSP-1 Cre mice (or
$\operatorname{Tgfbr} 2^{f s p K O / w t}$; heterozygous knockout), which were subsequently backcrossed to Tgfbr2 floxE2/floxE2 mice to produce Tgfbr $2^{\text {floxE2/floxE2}}$; FSP1-Cre mice (hereafter Tgfbr ${ }^{f_{s p K O}}$; homozygous knockout). The thyroid glands were removed for histological analysis at 4-6 weeks of age. Alternatively, because these mice die at about 8 weeks, the thyroid glands were transplanted into the renal subcapsular regions of syngeneic mice at about 4 weeks of age and removed at 26 and 52 weeks of age.

Tgfbr $2^{\text {floxE2/floxE2 }}$ mice were also crossed with TPO-Cre mice. The resultant Tgfbr $2^{\text {floxE2/wt }}$; TPO-Cre $\left(\mathrm{Tgfbr} 2^{t p o K O / w t}\right.$ ) mice were then bred with Tgfbr2 $2^{f l o x E 2 /}$ floxE2 mice to produce Tgfbr $2^{\text {floxE2/floxE2 }}$; FSP1-Cre mice (hereafter Tgfbr $2^{t p o K O}$ ). These mice were sacrificed for histological analysis of the thyroid glands 4, 26 and 52 weeks of age.

In both sets of the experiments, equal numbers of male and female mice were used.

\section{Genotyping}

Genotyping of offspring was performed by polymerase chain reaction (PCR) with tail tips DNA (REDExtractN-Amp ${ }^{\text {TM }}$ Tissue PCR KIT; Sigma Chemical Co., St. Louis, MO) that was amplified with the appropriate primer pairs. The primers used to detect the Cre recombinase gene were 5' - tat ttg gtt tag agt ttg gca aca-3' (forward) and 5'- aga gac gga aat cca tcg ctc g-3' (reverse) (A and B, respectively, in Fig.1), yielding a 352 bp PCR product (Fig. 2, upper). The primers $\mathrm{C}$ (5'- taa aca agg tcc gga gec ca-3' (forward)) and D (5' - act tct gca aga ggt ccc ct -3' (reverse)) were used to distinguish wild type (wt) and Tgfbr2 $2^{\text {floxE2/floxE2 }}$ mice, which produce 420 and 540 bp PCR products in wt and Tgfbr2 floxE2/floxE2 mice, respectively (Fig. 2, middle). Two other primers (5' - gta act cac tca tgc aaa gt -3 ' and 5 ' - tct cca ccc act act gca gga $t-3$ ') were also used to amplify the DNA fragment of TSH gene as a positive control. Thermocycling conditions consisted of 40 cycles of $30 \mathrm{sec}$ at $94{ }^{\circ} \mathrm{C}, 30$ sec at $55-57.5^{\circ} \mathrm{C}$, and $30 \mathrm{sec}$ at $72^{\circ} \mathrm{C}$.

\section{Detection of genomic recombination by PCR}

Genomic DNA was extracted from the thyroid tissues and tail tips using DNeasy Blood \& Tissue Kit (QIAGEN, Valencia, CA). Selected regions of the target genes were amplified and analyzed by PCR. The following primers were used to detect recombination (Fig. 1); primer E (forward): 5' - agg gat gaa tgg get tgc tt-3'and primer F (reverse): 5' - ctc acc tca gag cet gat 


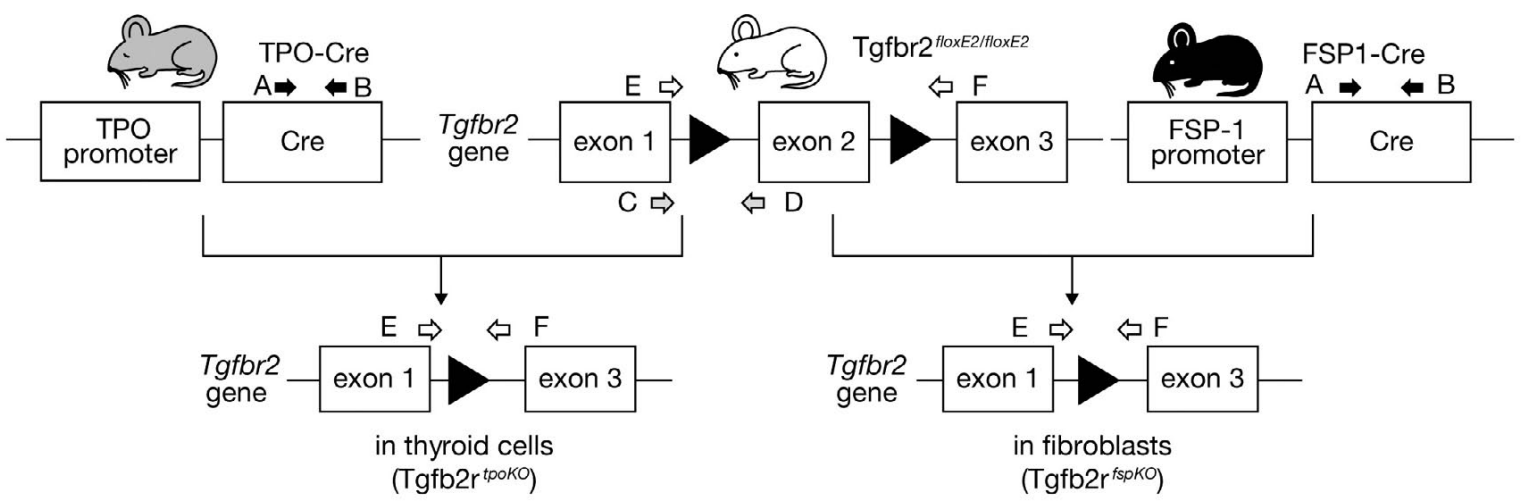

Fig. 1 Schematic representations of transgene structures in TPO-Cre, FSP1-Cre, Tgfbr $2^{\text {floxE2/floxE2 }}$, Tgfbr $2^{\text {tpoKO }}$ and Tgfbr2 ${ }^{f s p K O}$. The arrows indicate the primers used for genotyping.

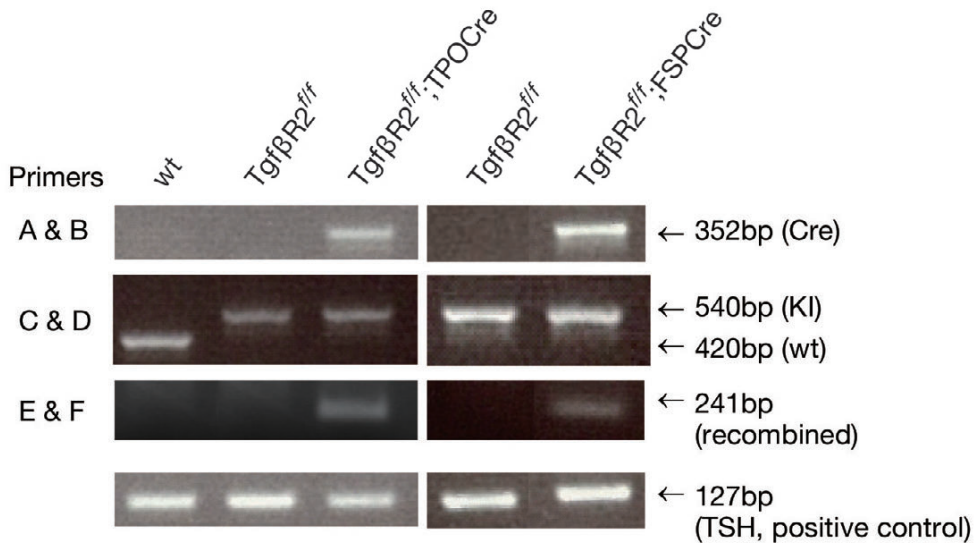

Fig. 2 PCR analysis of genotype and Cre-mediated DNA recombination. Genomic DNA was extracted from the tails or the thyroids and subjected to PCR analysis of genotyping (with the primers A \& B and C \& D) and Cre-mediated DNA recombination (with the primers E \& F) as described in the Materials and Methods. The locations of the primers A to F are shown in (A). Note that the primers A \& B amplify the 352 bp DNA fragment of Cre gene from TPO-Cre or FSP1-Cre mice, those of C \& D either the $540 \mathrm{bp}$ DNA fragment from Tgfbr floxE2/floxE2 or the $420 \mathrm{bp}$ DNA fragment from wt mice, and those of E \& F the $241 \mathrm{bp}$ recombined DNA fragment from Tgfb $2 \mathrm{r}^{t p o K O}$ or $\mathrm{Tgfb} 2 \mathrm{r}^{f s p K O}$. Two other primers were also used to amplify the TSH gene fragment as a positive control.

ta-3'. Primers E and F produce a 241 bp PCR product following recombination, while the distance of these two primers before recombination is $3.0 \mathrm{kbp}$.

\section{Free $T_{4}$ measurements}

Serum free $\mathrm{T}_{4}$ concentrations were measured by radioimmunoassay (RIA) as previously described [14]. The normal range was defined as the mean \pm 3 S.D. of untreated control mice.

$H \&$ E staining and immunohistochemistry (Ki67) of the thyroid glands

Thyroid tissues were fixed in formalin and embed- ded in paraffin. Five-micrometer-thick sections were prepared and stained with H\&E or immunostained with rabbit primary anti-Ki-67 antibody (Abcam, ab66155; a dilution of 1:100) and Alexa-fluor594-conjugaetd goat secondary anti-rabbit $\operatorname{IgG}(\mathrm{H}+\mathrm{L})$ antibody (Life Technologies, A11012; a dilution of 1:200). Nucleus was counter-stained with DAPI. Fluorescence was visualized by HS All-in-one Fluorescence Microscope BZ-9000 (Keyence). Results were expressed as cellular proliferation index (the numbers of Ki67-positive cells counted per those of all the cells counted (at least 500)). 


\section{Results}

Tgfbr $2^{t p o K O}$ mice which harbor the thyroid-specific disruption of $T g f b r 2$ gene was generated by crossing Tgfbr $2^{\text {floxE2/ffloxE2 }}$ and TPO-Cre mice (Fig. 1, left side). Successful recombination was confirmed by PCR amplification of the genomic DNA from the thyroid tissues, demonstrating the specific recombined DNA fragment (241 bp) of Tgfbr 2 gene in Tgfbr $2^{t p o K O}$ mice, not in wt or Tgfbr2 floxE2/floxE2 mice (Fig. 2). Similarly, Tgfbr $2^{f s p O}$ was generated with Tgfbr2 $2^{\text {floxE2/floxE2 }}$ and FSP-Cre mice (Fig. 1, right side), in which the recombined DNA fragment of the same size (241 bp) was amplified with the genomic DNA from their tail tips (Fig. 2).

Tgfbr $2^{t p o K O}$ mice grew normally and showed normal thyroid weights (data not shown) and normal thy- roid function at 26 and 52 weeks of ages, despite a slightly lower $\mathrm{T}_{4}$ levels at 4 weeks (Fig. 3), as compared to wt mice. During up to 52 week-follow up, all but one mice sacrificed at 4, 26 and 52 weeks ( $=15$ at each point) showed normal architectures without any tumor formation in thyroid histology (Fig. 4, left). One mouse exceptionally exhibited a benign follicular adenoma-like lesion in the entire region of the thyroid (Fig. 4, right) with a higher cellular proliferation index as compared to control mice ( $1.1 \%$ versus $0.59 \pm 0.15$ $\%($ mean \pm S.D., $n=3))$.

Tgfbr $2^{f s p K O}$ at 4 weeks of age also showed normal thyroid histology. Because these mice die at $\sim 8$ weeks, long-term observation could only be done after transplantation of their thyroid tissues into the syngeneic mice. Again, no abnormality was observed in all the thyroid tissues at 26 and 52 weeks of age $(n=4-6$ at

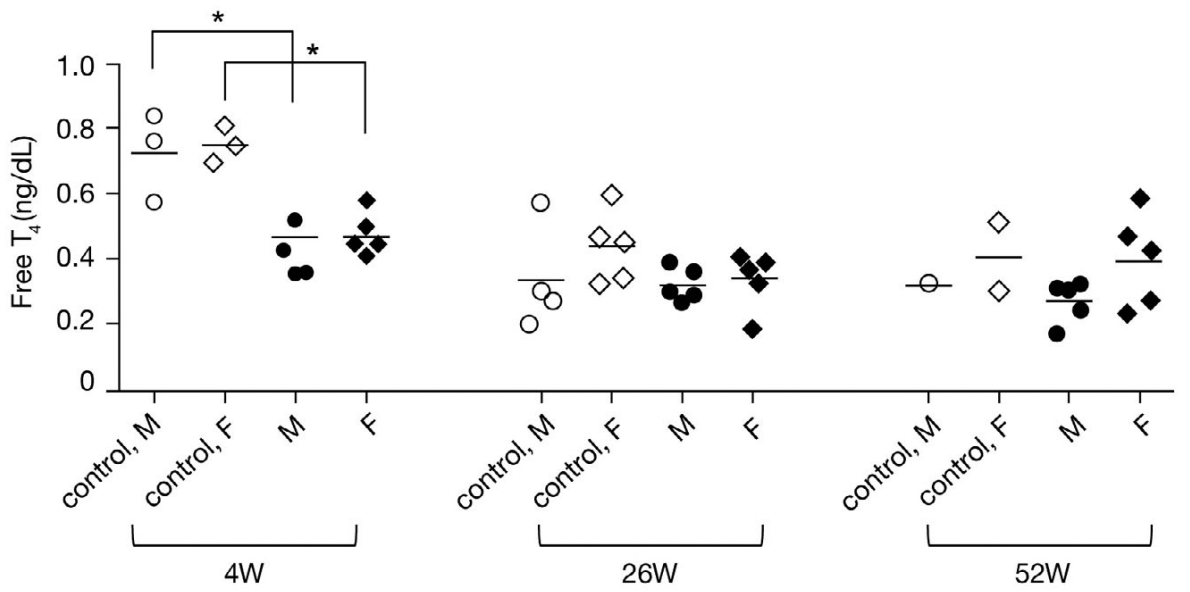

Fig. 3 Serum free $\mathrm{T}_{4}$ levels in wt and $\mathrm{Tgfb} 2 \mathrm{r}^{\text {tpoKO }}$ mice at 4,26 and 52 weeks of age. *, $p<0.05$.
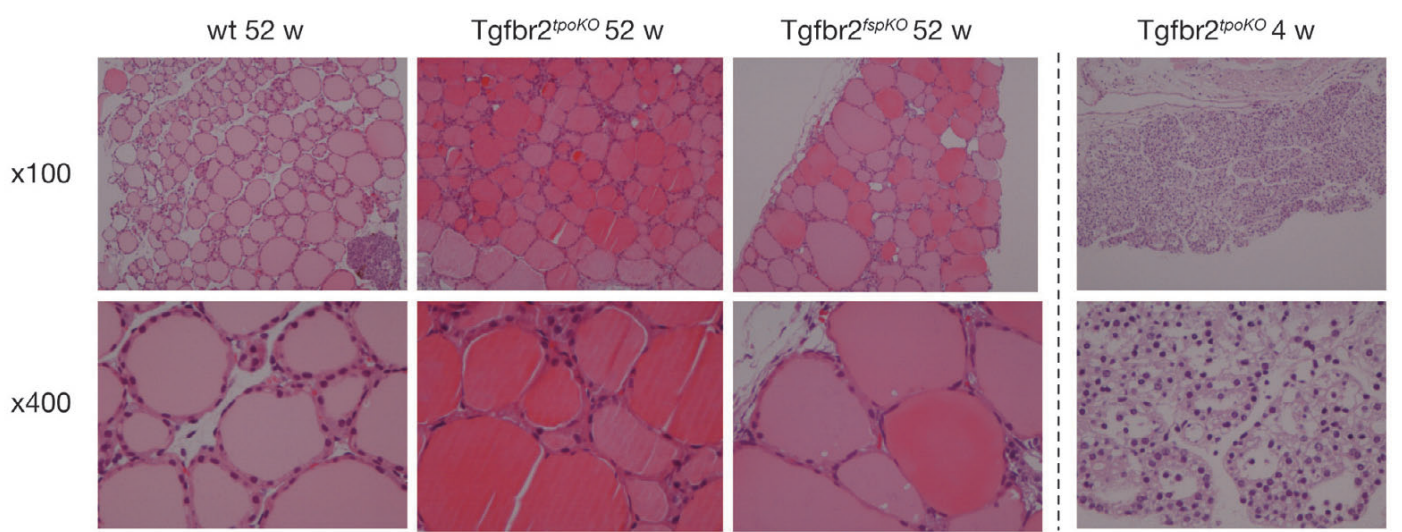

Fig. 4 Thyroid histology of $\operatorname{Tgfb} 2 \mathrm{r}^{t p o K O}$ and $\mathrm{Tgfb} 2 \mathrm{r}^{f s p K O}$ mice. The representative normal thyroid tissues of wt, Tfgb2 $\mathrm{r}^{t p o K O}$ and $\mathrm{Tfgb} 2 \mathrm{r}^{f s p K O}$ and a adenoma-like lesion in a Tgfb $2 \mathrm{r}^{\text {tpoKO }}$ mouse is shown. 
each point).

Thus, all the Tgfbr $2^{\text {tpoKO }}$ and $\mathrm{Tgfbr} 2^{f s p K O}$ mice exhibited normal thyroid structure except one of 15 Tgfbr $2^{t p o K O}$ mice developing a benign follicular adenoma-like lesion. Our results clearly demonstrate that a disruption of TGF- $\beta$ signaling by itself in the thyroid follicular epithelial cells or intrathyroidal fibroblasts does not have any noticeable effect on thyroid development and does not efficiently lead to thyroid tumor formation.

\section{Discussion}

It has long been well known that TGF- $\beta$ suppresses growth of normal thyroid follicular epithelial cells [11, 12]. However, the effect of TGF- $\beta$ on thyroid cancer cell behavior is controversial. Thus, some studies showed suppressive effect of TGF- $\beta$ on thyroid cancer cell proliferation $[15,16]$, whereas others resistance of thyroid cancers to TGF- $\beta$ 's anti-proliferative effect, at least partly due to miR-146b-5p mediated suppression of Smad4 expression [17], Smad4 mutations [18], elevated expression of Smad7 (an counterregulatory molecule of the canonical TGF- $\beta$ signaling) [19], down-regulation of T $\beta R I I$ expression [20], etc. However, the recent studies showing overexpression of TGF- $\beta$ in thyroid cancers of human and mouse origins [19, 21-25], particularly at their invasive fronts $[22,23,25]$, strongly indicate involvement of TGF- $\beta$ in invasion, metastasis and also epithelial-mesenchymal transition (EMT). From all these previous data as well as those with Tgfbr $2^{\text {tpoKO }}$ mice in the current study, it can be concluded as follows; TGF- $\beta$ does suppress proliferation of normal thyroid cells but is not strongly involved in tumor suppression by itself in the early carcinogenesis, and once thyroid cells transform, although some lose the responsiveness to TGF- $\beta$, TGF- $\beta$ acts as a tumor promoter by promoting the aggressive phenotype (invasive and metastatic capabilities, and EMT phenotype) in the majority of thyroid cancers.
The role(s) of TGF- $\beta$ signaling in fibroblasts have not extensively been studied in thyroid cancer. One paper has reported an association between higher expression of TGF- $\beta 1$ in thyroid cancer cells and increased $\alpha$-smooth muscle actin ( $\alpha$ SMA) levels in surrounding fibroblasts (an activation marker for cancer-associated fibroblasts), suggesting activation of fibroblasts by TGF- $\beta 1$ secreted from established cancer cells in a paracrine manner [21]. However, unlike prostate, stomach and mammary gland $[4,6,7]$, our results with $\mathrm{Tgfbr} 2^{f s p K O}$ mice demonstrate that a defect in TGF- $\beta$ signaling in intrathyroidal fibroblasts is not tumorigenic. Although the reason(s) for these different responses in distinct tissues are unknown, TGF- $\beta$ may be much less significant for early carcinogenesis or functional compensation by other members of the TGF- $\beta$ family such as bone morphogenetic proteins and activin may be more robust in some tissues like thyroids. It has been previously demonstrated enhancement of xenograft tumor growth by co-injection of rat thyroid cancer cells and fibroblasts in subcutaneous and orthotropic rat models [26], but the molecule(s) involved in this phenomenon was not determined.

In conclusion, our results indicate that TGF- $\beta$ signaling in epithelial or mesenchymal cells in the thyroid does not appear to function as a tumor suppressor at the early stage of carcinogenesis. Future studies will be necessary to elucidate the effect of a loss of TGF- $\beta$ signaling pathway on oncogene-induced carcinogenesis.

\section{Acknowledgements}

We thank Dr. Moses HL (Vanderbilt University) and Dr. Kimura S (NIH) for kind gifts of Tgfbr $2^{\text {floxE2/ffoxE2 }}$ and FSP1-Cre, and TPO-Cre mice, respectively.

\section{Disclosure}

None of the authors have any potential conflicts of interest associated with this research.

\section{References}

1. Attisano L, Wrana JL (2002) Signal transduction by the TGF-beta superfamily. Science 296: 1646-1647.

2. ten Dijke P, Hill CS (2004) New insights into TGF-betaSmad signalling. Trends Biochem Sci 29: 265-273.

3. Pickup M, Novitskiy S, Moses HL (2013) The roles of TGFbeta in the tumour microenvironment. Nat Rev
Cancer 13: 788-799.

4. Bhowmick NA, Chytil A, Plieth D, Gorska AE, Dumont $\mathrm{N}$, et al. (2004) TGF-beta signaling in fibroblasts modulates the oncogenic potential of adjacent epithelia. Science 303: 848-851.

5. Li X, Placencio V, Iturregui JM, Uwamariya C, Sharif- 
Afshar AR, Koyama T, et al. (2008) Prostate tumor progression is mediated by a paracrine TGF-beta/Wnt3a signaling axis. Oncogene 27: 7118-7130.

6. Cheng N, Bhowmick NA, Chytil A, Gorksa AE, Brown KA, et al. (2005) Loss of TGF-beta type II receptor in fibroblasts promotes mammary carcinoma growth and invasion through upregulation of TGF-alpha-, MSPand HGF-mediated signaling networks. Oncogene 24: 5053-5068.

7. Cheng N, Chytil A, Shyr Y, Joly A, Moses HL (2007) Enhanced hepatocyte growth factor signaling by type II transforming growth factor-beta receptor knockout fibroblasts promotes mammary tumorigenesis. Cancer Res 67: 4869-4877.

8. Forrester E, Chytil A, Bierie B, Aakre M, Gorska AE, et al. (2005) Effect of conditional knockout of the type II TGF-beta receptor gene in mammary epithelia on mammary gland development and polyomavirus middle $\mathrm{T}$ antigen induced tumor formation and metastasis. Cancer Res 65: 2296-2302.

9. Trobridge P, Knoblaugh S, Washington MK, Munoz NM, Tsuchiya KD, et al. (2009) TGF-beta receptor inactivation and mutant Kras induce intestinal neoplasms in mice via a beta-catenin-independent pathway. Gastroenterology 136: 1680-1688.e7.

10. Munoz NM, Upton M, Rojas A, Washington MK, Lin L, Chytil A, et al. (2006) Transforming growth factor beta receptor type II inactivation induces the malignant transformation of intestinal neoplasms initiated by Apc mutation. Cancer Res 66: 9837-9844.

11. Pisarev MA, Thomasz L, Juvenal GJ (2009) Role of transforming growth factor beta in the regulation of thyroid function and growth. Thyroid 19: 881-892.

12. Mincione G, Di Marcantonio MC, Tarantelli C, D'Inzeo S, Nicolussi A, et al. (2011) EGF and TGF-beta1 Effects on Thyroid Function. $J$ Thyroid Res 2011: 431718.

13. Kusakabe T, Kawaguchi A, Kawaguchi R, Feigenbaum L, Kimura S (2004) Thyrocyte-specific expression of Cre recombinase in transgenic mice. Genesis 39: 212216.

14. Nakahara M, Mitsutake N, Sakamoto H, Chen CR, Rapoport B, et al. (2010) Enhanced response to mouse thyroid-stimulating hormone (TSH) receptor immunization in TSH receptor-knockout mice. Endocrinology 151:4047-4054.

15. Turco A, Coppa A, Aloe S, Baccheschi G, Morrone S, et al. (1999) Overexpression of transforming growth factor beta-type II receptor reduces tumorigenicity and metastastic potential of K-ras-transformed thyroid cells. Int $J$ Cancer 80: 85-91.

16. Holting T, Zielke A, Siperstein AE, Clark OH, Duh QY (1994) Transforming growth factor-beta 1 is a negative regulator for differentiated thyroid cancer: studies of growth, migration, invasion, and adhesion of cultured follicular and papillary thyroid cancer cell lines. J Clin Endocrinol Metab 79: 806-813.

17. Geraldo MV, Fuziwara CS, Friguglieti CU, Costa RB, Kulcsar MA, et al. (2012) MicroRNAs miR-146-5p and let-7f as prognostic tools for aggressive papillary thyroid carcinoma: a case report. Arq Bras Endocrinol Metabol 56: 552-557.

18. Lazzereschi D, Nardi F, Turco A, Ottini L, D’Amico C, et al. (2005) A complex pattern of mutations and abnormal splicing of Smad4 is present in thyroid tumours. Oncogene 24: 5344-5354.

19. Matsuo SE, Fiore AP, Siguematu SM, Ebina KN, Friguglietti CU, et al. (2010) Expression of SMAD proteins, TGF-beta/activin signaling mediators, in human thyroid tissues. Arq Bras Endocrinol Metabol 54: 406412.

20. Lazzereschi D, Ranieri A, Mincione G, Taccogna S, Nardi F, et al. (1997) Human malignant thyroid tumors displayed reduced levels of transforming growth factor beta receptor type II messenger RNA and protein. Cancer Res 57: 2071-2076.

21. Zhang J, Wang Y, Li D, Jing S (2013) Notch and TGFbeta/Smad3 pathways are involved in the interaction between cancer cells and cancer-associated fibroblasts in papillary thyroid carcinoma. Tumour Biol [Epub ahead of print].

22. Eloy C, Santos J, Cameselle-Teijeiro J, Soares P, Sobrinho-Simoes M (2012) TGF-beta/Smad pathway and BRAF mutation play different roles in circumscribed and infiltrative papillary thyroid carcinoma. Virchows Arch 460: 587-600.

23. Riesco-Eizaguirre G, Rodriguez I, De la Vieja A, Costamagna E, Carrasco N, et al. (2009) The BRAFV600E oncogene induces transforming growth factor beta secretion leading to sodium iodide symporter repression and increased malignancy in thyroid cancer. Cancer Res 69: 8317-8325.

24. Knauf JA, Sartor MA, Medvedovic M, Lundsmith E, Ryder M, et al. (2011) Progression of BRAF-induced thyroid cancer is associated with epithelial-mesenchymal transition requiring concomitant MAP kinase and TGFbeta signaling. Oncogene 30: 3153-3162.

25. Vasko V, Espinosa AV, Scouten W, He H, Auer H, et al. (2007) Gene expression and functional evidence of epithelial-to-mesenchymal transition in papillary thyroid carcinoma invasion. Proc Natl Acad Sci USA 104: 2803-2808.

26. Saitoh O, Mitsutake N, Nakayama T, Nagayama Y (2009) Fibroblast-mediated in vivo and in vitro growth promotion of tumorigenic rat thyroid carcinoma cells but not normal Fisher rat thyroid follicular cells. Thyroid 19: 735-742. 